\title{
MULTIPLE SOLUTIONS TO NONLINEAR SCHRÖDINGER EQUATIONS WITH SINGULAR ELECTROMAGNETIC POTENTIAL
}

\author{
MÓNICA CLAPP AND ANDRZEJ SZULKIN
}

To Kazik Gęba on the occasion of his birthday, with friendship and great esteem.

ABStRaCt. We consider the semilinear electromagnetic Schrödinger equation

$$
(-i \nabla+\mathcal{A}(x))^{2} u+V(x) u=|u|^{2^{*}-2} u, \quad u \in D_{\mathcal{A}, 0}^{1,2}(\Omega, \mathbb{C}),
$$

where $\Omega=\left(\mathbb{R}^{m} \backslash\{0\}\right) \times \mathbb{R}^{N-m}$ with $2 \leq m \leq N, N \geq 3,2^{*}:=\frac{2 N}{N-2}$ is the critical Sobolev exponent, $V$ is a Hardy term and $\mathcal{A}$ is a singular magnetic potential of a particular form which includes the Aharonov-Bohm potentials. Under some symmetry assumptions on $\mathcal{A}$ we obtain multiplicity of solutions satisfying certain symmetry properties.

2010 Mathematics Subject Classification: Primary 35Q55; Secondary 35J61.

KEywords: Nonlinear Schrödinger equation, electromagnetic potential, Aharonov-Bohm potential, gauge invariance, symmetry properties.

\section{INTRODUCTION}

Our purpose is to extend some results contained in [1] and [7. We consider the semilinear electromagnetic Schrödinger problem

$$
\left\{\begin{array}{l}
(-i \nabla+\mathcal{A}(x))^{2} u+V(x) u=|u|^{2^{*}-2} u \\
u \in D_{\mathcal{A}, 0}^{1,2}(\Omega, \mathbb{C})
\end{array}\right.
$$

where $\Omega$ is a domain in $\mathbb{R}^{N}, N \geq 3,2^{*}:=\frac{2 N}{N-2}$ is the critical Sobolev exponent, $(-i \nabla+\mathcal{A})^{2}+V$ is the Hamiltonian for a (non-relativistic) charged particle in an electromagnetic field, $\mathcal{A}$ is the magnetic (or vector) potential and $V$ the electric (or scalar) potential for this field. The space $D_{\mathcal{A}, 0}^{1,2}(\Omega, \mathbb{C})$ is the closure of $C_{c}^{\infty}(\Omega, \mathbb{C})$ with respect to the norm

$$
\|u\|_{\mathcal{A}}:=\left(\int_{\Omega}\left|\nabla_{\mathcal{A}} u\right|^{2}\right)^{1 / 2}
$$

where $\nabla_{\mathcal{A}} u:=\nabla u+i \mathcal{A} u$. If $\Omega=\mathbb{R}^{N}$, we suppress the index 0 and write $D_{\mathcal{A}}^{1,2}\left(\mathbb{R}^{N}, \mathbb{C}\right)$.

Recall that if $\mathcal{A}=\left(\mathcal{A}_{1}, \ldots, \mathcal{A}_{N}\right)$ is a vector field defined in some open subset $\Omega$ of $\mathbb{R}^{N}$, then $\operatorname{curl} \mathcal{A}$ is the $N \times N$ skew-symmetric matrix $\mathcal{B}$ with entries $\mathcal{B}_{j k}=\partial_{j} \mathcal{A}_{k}-\partial_{k} \mathcal{A}_{j}$, or in the language of differential forms, if $\mathcal{A}$ is the 1 -form $\mathcal{A}_{1} d x_{1}+\cdots+\mathcal{A}_{N} d x_{N}$, then $\mathcal{B}=d \mathcal{A}$. If $\Omega$ is simply connected and $\operatorname{curl} \mathcal{A}=0$ then $\mathcal{A}=\nabla \varphi$ for some $\varphi$.

In what follows $\mathcal{A}$ will be singular and $V$ will be a Hardy-type potential.

The research of Mónica Clapp was partially supported by CONACYT grant 129847 and UNAM-DGAPA-PAPIIT grant IN106612 (Mexico).

The research of Andrzej Szulkin was partially supported by a grant from Swedish Research Council. 
As usual, we denote by $O(m)$ the group of linear isometries of $\mathbb{R}^{m}$ and by $S O(m)$ the subgroup of those whose determinant is 1 . We identify $S O(2)$ with the group of unit complex numbers $\mathbb{S}^{1}$ acting by multiplication on $\mathbb{C} \equiv \mathbb{R}^{2}$.

We start by considering a problem with a point singularity:

$$
\left\{\begin{array}{l}
(-i \nabla+\mathcal{A})^{2} u-\frac{\sigma}{|x|^{2}} u=|u|^{2^{*}-2} u, \\
u \in D_{\mathcal{A}, 0}^{1,2}(\Omega, \mathbb{C}),
\end{array}\right.
$$

where $\Omega:=\mathbb{R}^{N} \backslash\{0\}$ and $\mathcal{A}$ is of the form

$$
\mathcal{A}(x)=\frac{\widetilde{\mathcal{A}}(\omega)}{|x|}, \quad \omega:=\frac{x}{|x|} \quad \text { and } \quad \widetilde{\mathcal{A}} \in L^{\infty}\left(\mathbb{S}^{N-1}, \mathbb{R}^{N}\right) .
$$

We fix a closed subgroup $G$ of $O(N)$ and a continuous homomorphism of groups $\tau: G \rightarrow \mathbb{S}^{1}$, and denote by $G x:=\{g x: g \in G\}$ the $G$-orbit of a point $x \in \mathbb{R}^{N}$ and by $G_{x}:=\{g \in G: g x=x\}$ its isotropy group. We write \#Gx for the cardinality of $G x$ and $\operatorname{ker} \tau$ for the kernel of $\tau$. We assume that $\mathcal{A}$ is $G$-equivariant, that is,

$$
\mathcal{A}(g x)=g \mathcal{A}(x) \quad \text { for all } x \in \Omega, g \in G \text {. }
$$

We prove the following result.

Theorem 1.1. Assume that $\# G x=\infty$ for every $x \in \mathbb{R}^{N} \backslash\{0\}$ and that there exists $x^{*} \in \mathbb{R}^{N}$ such that $G_{x^{*}} \subset \operatorname{ker} \tau$. Then, if $\mathcal{A}$ satisfies (1.3), (1.4) and $\sigma<\left(\frac{N-2}{2}\right)^{2}$, there exists a nontrivial solution $u$ of problem (1.2) such that

$$
u(g x)=\tau(g) u(x) \quad \forall g \in G, \forall x \in \Omega .
$$

Recall that $\left(\frac{N-2}{2}\right)^{2}$ is the Hardy constant. Theorem 1.1 is a special case of a result for more general singular vector potentials which we state later (cf. Theorem 3.1).

A function $u$ satisfying (1.5) will be called $\tau$-equivariant. If $\tau \equiv 1$ is the trivial homomorphism then (1.5) simply says that $u$ is G-invariant, i.e.

$$
u(g x)=u(x) \quad \forall g \in G, \forall x \in \Omega .
$$

Condition (1.5) implies that the absolute value $|u|$ of $u$ is $G$-invariant, whereas the phase of $u(g x)$ is that of $u(x)$ multiplied by $\tau(g)$.

The special case where $G=\mathbb{S}^{1} \times O(N-2), N \geq 4$, and $\tau=\tau_{n}$ with $\tau_{n}\left(e^{i \vartheta}, h\right):=e^{i n \vartheta}, n \in \mathbb{Z}$, was considered by Abatangelo and Terracini in [1]. Note that, if $u_{n}$ satisfies (1.5) for this data and $u_{n}(y, z) \neq 0$ for some $y \in \mathbb{R}^{2} \backslash\{0\} \equiv \mathbb{C} \backslash\{0\}, z \in \mathbb{R}^{N-2}$, then

$$
u_{n}\left(e^{i \vartheta} y, z\right)=e^{i n \vartheta} u_{n}(y, z) \neq 0 \text { for all } \vartheta \in[0,2 \pi)
$$

i.e. $u_{n}$ restricted to the circle $\mathbb{S}^{1}(y, z):=\left\{\left(e^{i \vartheta} y, z\right): \vartheta \in[0,2 \pi)\right\}$ has degree $n$ (as a mapping into $\mathbb{C} \backslash\{0\})$. Therefore, if all $u_{n}$ are nontrivial a.e., then $u_{m} \neq u_{n}$ whenever $m \neq n$. So, in this case, Theorem 1.1 gives us infinitely many distinct solutions $u_{n}, n \in \mathbb{Z}$, having the symmetries we just described. 
For Aharonov-Bohm potentials we get a stronger result. Again, we write a point in $\mathbb{R}^{N}$ as $(y, z) \in \mathbb{R}^{2} \times \mathbb{R}^{N-2}$ and consider the problem

$$
\left\{\begin{array}{l}
(-i \nabla+s \mathbb{A})^{2} u-\frac{\sigma}{|y|^{2}} u=|u|^{2^{*}-2} u, \\
u \in D_{s \mathbb{A}, 0}^{1,2}(\Omega, \mathbb{C}),
\end{array}\right.
$$

where $\Omega:=\left(\mathbb{R}^{2} \backslash\{0\}\right) \times \mathbb{R}^{N-2} \equiv(\mathbb{C} \backslash\{0\}) \times \mathbb{R}^{N-2}, N \geq 3, s, \sigma \in \mathbb{R}$, and $\mathbb{A}: \Omega \rightarrow \mathbb{R}^{N}$ is the Aharonov-Bohm magnetic potential

$$
\mathbb{A}\left(y_{1}, y_{2}, z\right):=\frac{1}{|y|^{2}}\left(-y_{2}, y_{1}, 0\right), \quad y=\left(y_{1}, y_{2}\right) .
$$

We assume that $\sigma<\kappa_{s}$, where $\kappa_{s}$ is the best constant for the Hardy inequality

$$
\kappa_{s} \int_{\Omega} \frac{|\varphi|^{2}}{|y|^{2}} \leq \int_{\Omega}|\nabla \varphi+i s \mathbb{A} \varphi|^{2} \quad \forall \varphi \in C_{c}^{\infty}(\Omega, \mathbb{C}) .
$$

We prove the following result.

Theorem 1.2. If $\sigma<\kappa_{s}$, then for every $n \in \mathbb{Z}$ there exists a nontrivial solution $u_{n}$ of problem $\wp$ with the following properties:

(a) $u_{0}=\left|u_{0}\right|$ and $\left|u_{n}\right|>0$ in $\Omega$,

(b) $\left|u_{n}\right| \neq\left|u_{m}\right|$ if $|s+m| \neq|s+n|$,

(c) $u_{n}\left(e^{i \vartheta} y, z\right)=e^{i n \vartheta} u_{n}(y, z)$ for all $e^{i \vartheta} \in \mathbb{S}^{1},(y, z) \in \Omega$,

(d) $u_{n}(y, h z)=u_{n}(y, z)$ for all $h \in O(N-2),(y, z) \in \Omega$,

(e) $\left\|u_{m}\right\|_{s \mathbb{A}, \sigma}<\left\|u_{n}\right\|_{s \mathbb{A}, \sigma}$ if $|s+m|<|s+n|$.

For $N=3$ and $\sigma=0$ this result was proved in 7 . Properties (c) and (d) can be expressed by saying that $u_{n}$ satisfies (1.5) for $G:=\mathbb{S}^{1} \times O(N-2)$ and $\tau=\tau_{n}$, as defined above.

Existence of $\tau$-equivariant solutions can be obtained by constrained minimization in some appropriate function space, see e.g. [5. This is how we proceed to prove Theorem 1.1. For Theorem 1.2 we give a different argument: we obtain an $\mathbb{S}^{1}$-invariant solution by minimization and then use the gauge invariance of the Aharonov-Bohm potential to produce the other solutions. This allows us to establish not only existence and the symmetry properties, but also all other properties stated in Theorem 1.2

Our Theorems 1.1 and 1.2 extend Theorem 1.1 in [1. While in [1] it is assumed that $|\sigma|$ is sufficiently large and $\sigma<0$, we allow all $\sigma<\left(\frac{N-2}{2}\right)^{2}$ and $\sigma<\kappa_{s}$ respectively. We also allow more general symmetries and our arguments are considerably simpler. On the other hand, in [1 it was shown that, for $|\sigma|$ and $n$ large enough, there also exists a solution which satisfies (1.5) with $\tau=\tau_{n}$ for all $g \in \mathbb{Z} / n \times O(N-2)$ but not for all $g \in \mathbb{S}^{1} \times O(N-2)$. This does not follow from our results.

Note that the Hardy term in (1.2) is $S O(N)$-invariant. A natural question arises whether there exist meaningful $S O(N)$-equivariant magnetic potentials. The answer is contained in the next result.

Theorem 1.3. Let $\Omega \subset \mathbb{R}^{N}$ be a radially symmetric domain and $\mathcal{A} \in L_{\text {loc }}^{2}\left(\Omega, \mathbb{R}^{N}\right), N \geq 3$.

(i) If $\mathcal{A}(g x)=g \mathcal{A}(x)$ for all $g \in S O(N), x \in \Omega$, then $\mathcal{A}(x)=\nu(|x|) x$ for some real-valued function $\nu$. In particular, $\mathcal{A}(x)=\nabla \varphi(x)$ for some $S O(N)$-invariant $\varphi \in W_{\text {loc }}^{1,2}(\Omega, \mathbb{R})$. 
(ii) If $\mathcal{B}=\operatorname{curl} \mathcal{A}$ (in the sense of distributions) for some $\mathcal{A} \in L_{\text {loc }}^{2}\left(\Omega, \mathbb{R}^{N}\right)$ and $\mathcal{B}(g x)=$ $g \mathcal{B}(x) g^{-1}$ for all $g \in S O(N), x \in \Omega$, then $\mathcal{B}=0$.

A consequence of this theorem is that such $\mathcal{A}$ can be gauged away by an $S O(N)$-invariant gauge transformation, so the problem may be reduced to a non-magnetic one. More precisely, there is a one-to-one correspondence between radially symmetric solutions to (1.1) with $\mathcal{A}$ as above and to (1.1) with $\mathcal{A}=0$. We also give an example showing that radial solutions to (1.1) may exist even if $\mathcal{A}$ is not $S O(N)$-equivariant.

This paper is organized as follows: Section 2 contains preliminary material. In section 3 we state and prove an extension of Theorem 1.1 to more general singular magnetic potentials. Section 4 4 is devoted to the proof of Theorem 1.2 and Theorem 1.3 is proved in section 5

\section{Preliminaries}

Let $2 \leq m \leq N$. A point in $\mathbb{R}^{N}$ will be written as $(y, z) \in \mathbb{R}^{m} \times \mathbb{R}^{N-m}$. In the following we assume that

$$
\Omega=\left(\mathbb{R}^{m} \backslash\{0\}\right) \times \mathbb{R}^{N-m}
$$

and $\mathcal{A}$ is of the form

$$
\mathcal{A}(y, z)=\frac{\widetilde{\mathcal{A}}(\omega, z)}{|y|} \quad \text { with } \omega:=\frac{y}{|y|} \quad \text { and } \quad \widetilde{\mathcal{A}} \in L^{\infty}\left(\mathbb{S}^{m-1} \times \mathbb{R}^{N-m}, \mathbb{R}^{N}\right),
$$

where $\mathbb{S}^{m-1}$ is the unit sphere in $\mathbb{R}^{m}$, centered at the origin. Note that $\mathcal{A}$ in (1.3) and $\mathbb{A}$ in (1.6) have this form, with $m=N$ and $m=2$ respectively.

2.1. Basic inequalities. If $u \in D_{\mathcal{A}, 0}^{1,2}(\Omega, \mathbb{C})$ then its absolute value $|u| \in D_{0}^{1,2}(\Omega, \mathbb{R})$ and

$$
|\nabla| u|(x)| \leq\left|\nabla_{\mathcal{A}} u(x)\right| \quad \text { for a.e. } x \in \mathbb{R}^{N} .
$$

This is called the diamagnetic inequality [12. Together with the Sobolev inequality it yields

$$
S\left(\int_{\Omega}|u|^{2^{*}}\right)^{2 / 2^{*}} \leq \int_{\Omega}\left|\nabla_{\mathcal{A}} u\right|^{2}
$$

where $S$ is the best Sobolev constant.

The generalized Hardy inequality [3] says that

$$
\left(\frac{m-2}{2}\right)^{2} \int_{\mathbb{R}^{N}} \frac{|v|^{2}}{|y|^{2}} \leq \int_{\mathbb{R}^{N}}|\nabla v|^{2} \quad \forall v \in D_{0}^{1,2}\left(\mathbb{R}^{N}, \mathbb{R}\right) .
$$

The constant $\left(\frac{m-2}{2}\right)^{2}$ is optimal [15]. Combining this inequality with the diamagnetic inequality we obtain the magnetic Hardy inequality

$$
\left(\frac{m-2}{2}\right)^{2} \int_{\Omega} \frac{|u|^{2}}{|y|^{2}} \leq \int_{\Omega}\left|\nabla_{\mathcal{A}} u\right|^{2} \quad \forall u \in D_{\mathcal{A}, 0}^{1,2}(\Omega, \mathbb{C}) .
$$

A consequence is that

$$
\|u\|_{\mathcal{A}, \sigma}:=\left(\int_{\Omega}\left|\nabla_{\mathcal{A}} u\right|^{2}-\frac{\sigma}{|y|^{2}}|u|^{2}\right)^{1 / 2}
$$

are equivalent norms in $D_{\mathcal{A}, 0}^{1,2}(\Omega, \mathbb{C})$ for all $\sigma<\left(\frac{m-2}{2}\right)^{2}$. 
If $m=2$ the inequality (2.5) becomes trivial. Yet for Aharonov-Bohm potentials there are nontrivial Hardy inequalities. Laptev and Weidl showed in [11 that $s \mathbb{A}$ in $\mathbb{R}^{2}$ satisfies

$$
\min _{k \in \mathbb{Z}}|k+s|^{2} \int_{\mathbb{R}^{2}} \frac{|\varphi|^{2}}{|y|^{2}} \leq \int_{\mathbb{R}^{2}}|\nabla \varphi+i s \mathbb{A} \varphi|^{2} \quad \forall \varphi \in C_{c}^{\infty}\left(\mathbb{R}^{2} \backslash\{0\}, \mathbb{C}\right) .
$$

Integrating this inequality with respect to $z$ we obtain the Hardy inequality for Aharonov-Bohm potentials

$$
\min _{k \in \mathbb{Z}}|k+s|^{2} \int_{\mathbb{R}^{N}} \frac{|\varphi|^{2}}{|y|^{2}} \leq \int_{\mathbb{R}^{N}}\left|\nabla_{s \mathbb{A}} \varphi\right|^{2} \quad \forall \varphi \in C_{c}^{\infty}(\Omega, \mathbb{C}) .
$$

It was shown in [1] that $\kappa_{s}:=\min _{k \in \mathbb{Z}}|k+s|^{2} \in[0,1 / 4]$ is the best constant for inequality (2.6). Again, a consequence is that

$$
\|u\|_{s \mathbb{A}, \sigma}:=\left(\int_{\Omega}\left|\nabla_{s \mathbb{A}} u\right|^{2}-\frac{\sigma}{|y|^{2}}|u|^{2}\right)^{1 / 2}
$$

are equivalent norms in $D_{s \mathbb{A}, 0}^{1,2}(\Omega, \mathbb{C})$ for all $\sigma<\kappa_{s}$.

Note that if $s \in \mathbb{Z}$ then $\kappa_{s}=0$ and the Hardy inequality (2.6) becomes trivial. We would like to point out that in 7 we claimed, incorrectly, that $\kappa_{1}>0$. However, we never used this fact.

Remark 2.1. Using (2.6) it was shown in [1, Section 5.2] that if $s \notin \mathbb{Z}$, then $D_{s \mathbb{A}, 0}^{1,2}(\Omega, \mathbb{C})$ is a proper subspace of $D^{1,2}\left(\mathbb{R}^{N}, \mathbb{C}\right)$ and the inclusion mapping is continuous. We point out that, if $s \in \mathbb{Z} \backslash\{0\}$, the situation is different: $D_{s \mathbb{A}, 0}^{1,2}(\Omega, \mathbb{C})$ and $D^{1,2}\left(\mathbb{R}^{N}, \mathbb{C}\right)$ are isometric but none of them is contained in the other. To see this, let $\theta=\theta(y, z)$ be the polar angle of $y$ in (1.6) and note that $\nabla \theta=\mathbb{A}$. Since $\mathbb{R}^{N} \backslash \Omega$ has capacity zero, $D^{1,2}\left(\mathbb{R}^{N}, \mathbb{C}\right)=D_{0}^{1,2}(\Omega, \mathbb{C})$ (cf. Theorem 4.7.2 in [9] and Theorem 9.2.3 in [13]). Now it follows easily that $u \mapsto e^{-i s \theta} u$ maps $D^{1,2}\left(\mathbb{R}^{N}, \mathbb{C}\right)$ isometrically onto $D_{s \mathbb{A}, 0}^{1,2}(\Omega, \mathbb{C})$. But if $\varphi \in C_{c}^{\infty}\left(\mathbb{R}^{N}, \mathbb{R}\right)$ is such that $\varphi(x)=1$ for $|x| \leq 1$, then $\varphi$ is in $D^{1,2}\left(\mathbb{R}^{N}, \mathbb{C}\right)$ but not in $D_{s \mathbb{A}, 0}^{1,2}(\Omega, \mathbb{C})$ because $1 /|y|^{2}$ is not locally integrable at 0 (in fact this shows that $D_{s \mathbb{A}, 0}^{1,2}(\Omega, \mathbb{C}) \neq D^{1,2}\left(\mathbb{R}^{N}, \mathbb{C}\right)$ for any $\left.s \neq 0\right)$. On the other hand, $e^{-i s \theta} \varphi$ is in $D_{s \mathbb{A}, 0}^{1,2}(\Omega, \mathbb{C})$ but not in $D^{1,2}\left(\mathbb{R}^{N}, \mathbb{C}\right)$.

2.2. The symmetries. Let $G$ be a closed subgroup of $O(N)$. We assume that $\Omega$ is $G$-invariant (i.e. $g x \in \Omega$ for all $x \in \Omega, g \in G)$. Since $\Omega=\left(\mathbb{R}^{m} \backslash\{0\}\right) \times \mathbb{R}^{N-m}$, this implies that $\{0\} \times \mathbb{R}^{N-m}$ is $G$-invariant and, therefore, $G$ must be a subgroup of $O(m) \times O(N-m)$. We also assume that $\mathcal{A}$ is $G$-equivariant (i.e. $\mathcal{A}(g x)=g \mathcal{A}(x)$ for all $x \in \Omega, g \in G$ ). We fix a continuous homomorphism $\tau: G \rightarrow \mathbb{S}^{1}$, and look for solutions which are $\tau$-equivariant, i.e.

$$
u(g x)=\tau(g) u(x) \quad \forall g \in G, \forall x \in \Omega .
$$

Example 2.2 (Aharonov-Bohm). If $G:=\mathbb{S}^{1} \times O(N-2)$, the Aharonov-Bohm potentials $s \mathbb{A}$ are $G$-equivariant. For each $n \in \mathbb{Z}$ we consider the homomorphism $\tau_{n}: G \rightarrow \mathbb{S}^{1}$ given by

$$
\tau_{n}\left(e^{i \vartheta}, h\right):=e^{i n \vartheta} \quad \text { for all } h \in O(N-2) .
$$

Note that $u$ is $\tau_{n}$-equivariant iff it satisfies properties (c) and (d) of Theorem 1.2 . 
Example 2.3 (Point singularity). A magnetic potential of the form

$$
\mathcal{A}(x)=\frac{\widetilde{\mathcal{A}}(\omega)}{|x|} \quad \text { with } \quad \omega:=\frac{x}{|x|}, \quad \widetilde{\mathcal{A}} \in L^{\infty}\left(\mathbb{S}^{N-1}, \mathbb{R}^{N}\right),
$$

is $G$-equivariant iff $\widetilde{\mathcal{A}}$ is $G$-equivariant, i.e. $\widetilde{\mathcal{A}}(g \omega)=g \widetilde{\mathcal{A}}(\omega)$ for all $\omega \in \mathbb{S}^{N-1}, g \in G$. The case where $G:=\mathbb{S}^{1} \times O(N-2)$ and $\tau=\tau_{n}$, as above, was considered in [1]. But $G$ and $\tau$ may be more general. For example, if $N=2 j+k$ and $\Gamma$ is a subgroup of $O(k)$, we may take $G:=\mathbb{S}^{1} \times \Gamma$, with $\mathbb{S}^{1}$ acting by multiplication on $\mathbb{C}^{j} \equiv \mathbb{R}^{2 j}$, and $\tau_{n}\left(e^{i \vartheta}, h\right):=e^{i n \vartheta}$ for all $h \in \Gamma$. Then $u$ is $\tau_{n}$-equivariant iff

$$
u\left(e^{i \vartheta} \xi_{1}, \ldots, e^{i \vartheta} \xi_{j}, h \xi^{\prime}\right)=e^{i n \vartheta} u\left(\xi_{1}, \ldots, \xi_{j}, \xi^{\prime}\right) \text { for all } \xi_{1}, \ldots, \xi_{j} \in \mathbb{C}, \xi^{\prime} \in \mathbb{R}^{k} .
$$

Or we may take $G:=\mathbb{S}^{1} \times \cdots \times \mathbb{S}^{1}$ (with $j$ factors) and $\tau_{n_{1}, \ldots, n_{j}}\left(e^{i \vartheta_{1}}, \ldots, e^{i \vartheta_{j}}, h\right):=e^{i n_{1} \vartheta_{1}} \cdots e^{i n_{j} \vartheta_{j}}$ to obtain solutions satisfying

$$
u\left(e^{i \vartheta_{1}} \xi_{1}, \ldots, e^{i \vartheta_{j}} \xi_{j}, h \xi^{\prime}\right)=e^{i\left(n_{1} \vartheta_{1}+\cdots+n_{j} \vartheta_{j}\right)} u\left(\xi_{1}, \ldots, \xi_{j}, \xi^{\prime}\right) \text { for all } \xi_{1}, \ldots, \xi_{j} \in \mathbb{C}, \xi^{\prime} \in \mathbb{R}^{k} .
$$

Solutions satisfying (2.7) may be obtained by choosing appropriate subspaces of $D_{\mathcal{A}, 0}^{1,2}(\Omega, \mathbb{C})$. To each $u \in D_{\mathcal{A}, 0}^{1,2}(\Omega, \mathbb{C}), g \in G$, we associate the function $g \cdot_{\tau} u \in D_{\mathcal{A}, 0}^{1,2}(\Omega, \mathbb{C})$ defined as follows:

$$
\left(g \cdot_{\tau} u\right)(x):=\tau(g) u\left(g^{-1} x\right), \quad x \in \Omega .
$$

Then

$$
\left\|g \cdot_{\tau} u\right\|_{\mathcal{A}, \sigma}=\|u\|_{\mathcal{A}, \sigma} \quad \text { and } \quad\left|g \cdot_{\tau} u\right|_{2^{*}}=|u|_{2^{*}},
$$

where $|\cdot|_{p}$ denotes the $L^{p}(\Omega, \mathbb{C})$-norm. So this gives isometric $G$-actions on $D_{\mathcal{A}, 0}^{1,2}(\Omega, \mathbb{C})$ and $L^{2^{*}}(\Omega, \mathbb{C})$. We write

$$
D_{\mathcal{A}, 0}^{1,2}(\Omega, \mathbb{C})^{\tau}:=\left\{u \in D_{\mathcal{A}, 0}^{1,2}(\Omega, \mathbb{C}): u(g x)=\tau(g) u(x) \quad \forall g \in G, \forall x \in \Omega\right\}
$$

for the fixed point set of this action, which is a closed subspace of $D_{\mathcal{A}, 0}^{1,2}(\Omega, \mathbb{C})$. If $\tau \equiv 1$ we write

$$
D_{\mathcal{A}, 0}^{1,2}(\Omega, \mathbb{C})^{G}:=\left\{u \in D_{\mathcal{A}, 0}^{1,2}(\Omega, \mathbb{C}): u(g x)=u(x) \quad \forall g \in G, \forall x \in \Omega\right\}
$$

instead of $D_{\mathcal{A}, 0}^{1,2}(\Omega, \mathbb{C})^{\tau}$.

Some of our solutions will be obtained by constrained minimization. If

$$
\inf _{\substack{u \in D_{\mathcal{A}, 0}^{1,2}(\Omega, \mathbb{C})^{\tau} \\ u \neq 0}} \frac{\|u\|_{\mathcal{A}, \sigma}^{2}}{|u|_{2^{*}}^{2}}=\inf \left\{\|u\|_{\mathcal{A}, \sigma}^{2}: u \in D_{\mathcal{A}, 0}^{1,2}(\Omega, \mathbb{C})^{\tau},|u|_{2^{*}}^{2}=1\right\}
$$

is attained at some $\bar{u}$ with $|\bar{u}|_{2^{*}}=1$, then it follows from the Lagrange multiplier rule and the principle of symmetric criticality [14, 18] that $u=\|\bar{u}\|_{\mathcal{A}, \sigma}^{2 /\left(2^{*}-2\right)} \bar{u}$ is a solution of (1.1) satisfying (2.7). Here it is important to make sure that $D_{\mathcal{A}, 0}^{1,2}(\Omega, \mathbb{C})^{\tau}$ is nontrivial. A necessary and sufficient condition for this is given in the following result.

Proposition 2.4. Let $G$ be a closed subgroup of $O(N), \tau: G \rightarrow \mathbb{S}^{1}$ a continuous homomorphism of groups and $\Omega \subset \mathbb{R}^{N}$ a G-invariant domain.

(i) If there exists $x^{*} \in \Omega$ such that $G_{x^{*}} \subset \operatorname{ker} \tau$, then $D_{\mathcal{A}, 0}^{1,2}(\Omega, \mathbb{C})^{\tau}$ is infinite dimensional.

(ii) If no $x^{*}$ as above exists, then $D_{\mathcal{A}, 0}^{1,2}(\Omega, \mathbb{C})^{\tau}=\{0\}$. 
Proof. (i) This result has been shown in [6], see the proof of Theorem 1.1 there. For the reader's convenience we include a (slightly different) argument. Recall that two groups $H, K \subset G$ are called conjugate in $G$ if $K=g^{-1} H g$ for some $g \in G$ and denote the conjugacy class of $H$ by $(H)$. By Theorem 5.14 in Chapter I of $[8]$ there exists a unique conjugacy class $(P), P \subset G$, such that the set

$$
\Omega_{(P)}:=\left\{x \in \mathbb{R}^{N}: G_{x} \text { is conjugate to } P \text { in } G\right\}
$$

is open and dense in $\Omega$. Moreover, if $x \in \Omega \backslash \Omega_{(P)}$, then $P$ is conjugate to a (proper) subgroup of $G_{x}$. Let $x^{*} \in \Omega_{(P)}$, let $N$ be an open $G$-invariant tubular neighborhood of $G_{x^{*}}$ in $\Omega$ and denote the fiber over $x^{*}$ in $N$ by $N_{x^{*}}$. Then $\operatorname{dim} N_{x^{*}}=N-\operatorname{dim} G_{x^{*}}>0$. For each $\varphi \in C_{c}^{\infty}\left(N_{x^{*}}, \mathbb{C}\right)$ we can now define

$$
\widetilde{\varphi}(g x):=\tau(g) \varphi(x), \quad x \in N_{x^{*}}, g \in G .
$$

Since $G_{x}$ is conjugate to $P, G_{x} \subset \operatorname{ker} \tau$ and it follows that $\widetilde{\varphi}$ is well defined. Clearly, the space of all $\widetilde{\varphi}$ as above is an infinite dimensional subspace of $C_{c}^{\infty}(\Omega, \mathbb{C})$.

(ii) If for each $x \in \Omega$ there exists $g \in G_{x}$ such that $\tau(g) \neq 1$, then $u(x)=u(g x)=\tau(g) u(x)$ and, hence, $u(x)=0$.

As an example that (ii) can occur we may take $G=O(N)$ and the determinant of $g$ as $\tau(g)$. Then for each $x \in \Omega$ there exists a $g_{x} \in O(N)$ with $g_{x} x=x$ and $\tau\left(g_{x}\right)=-1$.

2.3. Concentration-compactness. An essential tool in our proofs will be the concentrationcompactness lemma which we now formulate. Let $\mathcal{M}\left(\mathbb{R}^{N}\right)$ denote the space of finite measures in $\mathbb{R}^{N}$ and set

$$
S_{\mathcal{A}, \sigma}^{\tau}:=\inf _{\substack{u \in D_{\mathcal{A}, 0}^{1,2}(\Omega, \mathbb{C})^{\tau} \\ u \neq 0}} \frac{\|u\|_{\mathcal{A}, \sigma}^{2}}{|u|_{2^{*}}^{2}} .
$$

If $\tau \equiv 1$ we write $S_{\mathcal{A}, \sigma}^{G}$ instead of $S_{\mathcal{A}, \sigma}^{\tau}$.

Lemma 2.5 (Concentration-compactness). Let $\sigma<\left(\frac{m-2}{2}\right)^{2}$ or $\sigma<\kappa_{s}$ if $\mathcal{A}=s \mathbb{A}$, and let $\left(u_{n}\right)$ be a sequence in $D_{\mathcal{A}, 0}^{1,2}(\Omega, \mathbb{C})^{\tau}$ such that

$$
\begin{aligned}
u_{n} \rightarrow u & \text { weakly in } D_{\mathcal{A}, 0}^{1,2}(\Omega, \mathbb{C}), \\
\left|\nabla_{\mathcal{A}}\left(u_{n}-u\right)\right|^{2}-\frac{\sigma}{|y|^{2}}\left|u_{n}-u\right|^{2} \rightarrow \mu & \text { weakly in } \mathcal{M}\left(\mathbb{R}^{N}\right), \\
\left|u_{n}-u\right|^{2^{*}} \rightarrow \nu & \text { weakly in } \mathcal{M}\left(\mathbb{R}^{N}\right), \\
u_{n}(x) \rightarrow u(x) & \text { a.e. in } \mathbb{R}^{N} .
\end{aligned}
$$

Set

$$
\begin{gathered}
\mu_{\infty}:=\lim _{R \rightarrow \infty} \limsup _{n \rightarrow \infty} \int_{|x| \geq R}\left(\left|\nabla_{\mathcal{A}} u_{n}\right|^{2}-\frac{\sigma}{|y|^{2}}\left|u_{n}\right|^{2}\right) \\
\quad \text { and } \nu_{\infty}:=\lim _{R \rightarrow \infty} \limsup _{n \rightarrow \infty} \int_{|x| \geq R}\left|u_{n}\right|^{2^{*}} .
\end{gathered}
$$

Then, the following hold:

(a) $S_{\mathcal{A}, \sigma}^{\tau}\|\nu\|^{2 / 2^{*}} \leq\|\mu\|$.

(b) $S_{\mathcal{A}, \sigma}^{\tau} \nu_{\infty}^{2 / 2^{*}} \leq \mu_{\infty}$.

(c) $\lim \sup _{n \rightarrow \infty}\left\|u_{n}\right\|_{\mathcal{A}, \sigma}^{2}=\|u\|_{\mathcal{A}, \sigma}^{2}+\|\mu\|+\mu_{\infty}$. 
(d) $\lim \sup _{n \rightarrow \infty}\left|u_{n}\right|_{2^{*}}^{2^{*}}=|u|_{2^{*}}^{2^{*}}+\|\nu\|+\nu_{\infty}$.

(e) If $u=0$ and $S_{\mathcal{A}, \sigma}^{\tau}\|\nu\|^{2 / 2^{*}}=\|\mu\|$, then $\mu$ and $\nu$ are concentrated at a single finite $G$-orbit of $\mathbb{R}^{N}$ or are zero.

The proof uses the same argument as in [7] which, in turn, is a modification of that in [2], see also [17 where the concentration-compactness lemma for $G$-invariant functions was introduced. There are, however, two facts which were obvious in 7 but need to be verified here. If $\sigma>0$, then it is not clear that $\mu$ is a positive measure and that $\mu_{\infty}$ is well defined and nonnegative. We show this in the next lemma.

Lemma 2.6. $\mu_{\infty} \geq 0$ and $\mu$ is a positive measure.

Proof. The arguments below are adaptations of those in the Appendix of [16] (for $\mu_{\infty}$ ) and Lemma 2.3 in [4] (for $\mu$ ). Since the conclusions are obvious if $\sigma \leq 0$, we assume that $\sigma>0$.

First we show that $\mu$ is a positive measure. Let $\varphi \in C_{c}^{\infty}\left(\mathbb{R}^{N}, \mathbb{R}\right)$ be $G$-invariant, $\varphi \geq 0$, and put $\varphi_{\varepsilon}:=\left(\sqrt{\varphi+\varepsilon^{2}}-\varepsilon\right)^{2}(\varepsilon>0)$ and $v_{n}:=u_{n}-u$. Using the Hardy inequality (2.4) or (2.6) and the fact that $\sqrt{\varphi_{\varepsilon}}$ is differentiable we obtain

$$
\begin{aligned}
0 \leq & \int_{\mathbb{R}^{N}}\left(\left|\nabla_{\mathcal{A}}\left(\sqrt{\varphi_{\varepsilon}} v_{n}\right)\right|^{2}-\frac{\sigma}{|y|^{2}}\left|\sqrt{\varphi_{\varepsilon}} v_{n}\right|^{2}\right) \\
= & \int_{\mathbb{R}^{N}} \varphi_{\varepsilon}\left(\left|\nabla_{\mathcal{A}} v_{n}\right|^{2}-\frac{\sigma}{|y|^{2}}\left|v_{n}\right|^{2}\right)+2 \operatorname{Re} \int_{\mathbb{R}^{N}} \sqrt{\varphi_{\varepsilon}} \bar{v}_{n} \nabla_{\mathcal{A}} v_{n} \cdot \nabla\left(\sqrt{\varphi_{\varepsilon}}\right) \\
& +\int_{\mathbb{R}^{N}}\left|\nabla\left(\sqrt{\varphi_{\varepsilon}}\right)\right|^{2}\left|v_{n}\right|^{2} .
\end{aligned}
$$

Since $v_{n} \rightarrow 0$ in $L_{l o c}^{2}\left(\mathbb{R}^{N}, \mathbb{C}\right)$, the second and the third term on the right-hand side above go to 0 as $n \rightarrow \infty$. So

$$
\left\langle\mu, \varphi_{\varepsilon}\right\rangle=\lim _{n \rightarrow \infty} \int_{\mathbb{R}^{N}} \varphi_{\varepsilon}\left(\left|\nabla_{\mathcal{A}} v_{n}\right|^{2}-\frac{\sigma}{|y|^{2}}\left|v_{n}\right|^{2}\right) \geq 0 \quad \text { and } \quad\langle\mu, \varphi\rangle \geq 0
$$

because $\varphi_{\varepsilon} \rightarrow \varphi$ in $L^{\infty}\left(\mathbb{R}^{N}, \mathbb{R}\right)$ as $\varepsilon \rightarrow 0$. This proves that $\mu \geq 0$. 
Next, we show that $\mu_{\infty}$ is well defined and nonnegative. Let $\psi_{R} \in C^{\infty}\left(\mathbb{R}^{N},[0,1]\right)$ be radially symmetric and such that $\psi_{R}(x)=0$ for $|x| \leq R, \psi_{R}(x)=1$ for $|x| \geq R+1$. Then

$$
\begin{aligned}
& \limsup _{n \rightarrow \infty}\left\|u_{n}\right\|_{\mathcal{A}, \sigma}^{2}=\|u\|_{\mathcal{A}, \sigma}^{2}+\limsup _{n \rightarrow \infty}\left\|v_{n}\right\|_{\mathcal{A}, \sigma}^{2} \\
& =\|u\|_{\mathcal{A}, \sigma}^{2}+\lim _{R \rightarrow \infty} \limsup _{n \rightarrow \infty}\left(\int_{\mathbb{R}^{N}}\left(1-\psi_{R}^{2}\right)\left(\left|\nabla_{\mathcal{A}} v_{n}\right|^{2}-\frac{\sigma}{|y|^{2}}\left|v_{n}\right|^{2}\right)\right. \\
& \left.\quad+\int_{\mathbb{R}^{N}} \psi_{R}^{2}\left(\left|\nabla_{\mathcal{A}} v_{n}\right|^{2}-\frac{\sigma}{|y|^{2}}\left|v_{n}\right|^{2}\right)\right) \\
& =\|u\|_{\mathcal{A}, \sigma}^{2}+\lim _{R \rightarrow \infty}\left(\int_{\mathbb{R}^{N}}\left(1-\psi_{R}^{2}\right) d \mu+\limsup _{n \rightarrow \infty} \int_{\mathbb{R}^{N}} \psi_{R}^{2}\left(\left|\nabla_{\mathcal{A}} v_{n}\right|^{2}-\frac{\sigma}{|y|^{2}}\left|v_{n}\right|^{2}\right)\right) \\
& =\|u\|_{\mathcal{A}, \sigma}^{2}+\|\mu\|+\lim _{R \rightarrow \infty} \limsup _{n \rightarrow \infty} \int_{\mathbb{R}^{N}} \psi_{R}^{2}\left(\left|\nabla_{\mathcal{A}} v_{n}\right|^{2}-\frac{\sigma}{|y|^{2}}\left|v_{n}\right|^{2}\right) \\
& =\|u\|_{\mathcal{A}, \sigma}^{2}+\|\mu\|+\lim _{R \rightarrow \infty} \limsup _{n \rightarrow \infty} \int_{\mathbb{R}^{N}} \psi_{R}^{2}\left(\left|\nabla_{\mathcal{A}} u_{n}\right|^{2}-\frac{\sigma}{|y|^{2}}\left|u_{n}\right|^{2}\right),
\end{aligned}
$$

where the last equality follows from the fact that

$$
\lim _{R \rightarrow \infty} \int_{\mathbb{R}^{N}} \psi_{R}^{2}\left(\left|\nabla_{\mathcal{A}} u\right|^{2}-\frac{\sigma}{|y|^{2}}|u|^{2}\right)=0 .
$$

Since

$$
\int_{\mathbb{R}^{N}} \psi_{R}^{2}\left|\nabla_{\mathcal{A}} u_{n}\right|^{2} \leq \int_{|x|>R}\left|\nabla_{\mathcal{A}} u_{n}\right|^{2} \leq \int_{\mathbb{R}^{N}} \psi_{R-1}^{2}\left|\nabla_{\mathcal{A}} u_{n}\right|^{2}
$$

and

$$
\int_{\mathbb{R}^{N}} \psi_{R}^{2} \frac{\left|u_{n}\right|^{2}}{|y|^{2}} \leq \int_{|x|>R} \frac{\left|u_{n}\right|^{2}}{|y|^{2}} \leq \int_{\mathbb{R}^{N}} \psi_{R-1}^{2} \frac{\left|u_{n}\right|^{2}}{|y|^{2}}
$$

we have, setting $I_{R}\left(u_{n}\right):=\int_{\mathbb{R}^{N}}\left(\psi_{R-1}^{2}-\psi_{R}^{2}\right) \frac{\sigma}{|y|^{2}}\left|u_{n}\right|^{2}$,

$$
\begin{gathered}
\int_{\mathbb{R}^{N}} \psi_{R}^{2}\left(\left|\nabla_{\mathcal{A}} u_{n}\right|^{2}-\frac{\sigma}{|y|^{2}}\left|u_{n}\right|^{2}\right)-I_{R}\left(u_{n}\right) \leq \int_{|x|>R}\left(\left|\nabla_{\mathcal{A}} u_{n}\right|^{2}-\frac{\sigma}{|y|^{2}}\left|u_{n}\right|^{2}\right) \\
\leq \int_{\mathbb{R}^{N}} \psi_{R-1}^{2}\left(\left|\nabla_{\mathcal{A}} u_{n}\right|^{2}-\frac{\sigma}{|y|^{2}}\left|u_{n}\right|^{2}\right)+I_{R}\left(u_{n}\right) .
\end{gathered}
$$

Since $u_{n} \rightarrow u$ in $L_{l o c}^{2}\left(\mathbb{R}^{N}, \mathbb{C}\right), \lim _{R \rightarrow \infty} \lim _{n \rightarrow \infty} I_{R}\left(u_{n}\right)=0$. So (2.10) implies that

$$
\begin{aligned}
\mu_{\infty}:= & \lim _{R \rightarrow \infty} \limsup _{n \rightarrow \infty} \int_{|x|>R}\left(\left|\nabla_{\mathcal{A}} u_{n}\right|^{2}-\frac{\sigma}{|y|^{2}}\left|u_{n}\right|^{2}\right) \\
& =\lim _{R \rightarrow \infty} \limsup _{n \rightarrow \infty} \int_{\mathbb{R}^{N}} \psi_{R}^{2}\left(\left|\nabla_{\mathcal{A}} u_{n}\right|^{2}-\frac{\sigma}{|y|^{2}}\left|u_{n}\right|^{2}\right) .
\end{aligned}
$$

Since the right-hand side above is well defined according to (2.9), also $\mu_{\infty}$ is well defined. Note that we have also proved that $\mu_{\infty}$ satisfies (c) of the lemma. 
Finally, similarly as in (2.8), we obtain

$$
\begin{aligned}
0 & \leq \int_{\mathbb{R}^{N}}\left(\left|\nabla_{\mathcal{A}}\left(\psi_{R} u_{n}\right)\right|^{2}-\frac{\sigma}{|y|^{2}}\left|\psi_{R} u_{n}\right|^{2}\right) \\
& =\int_{\mathbb{R}^{N}} \psi_{R}^{2}\left(\left|\nabla_{\mathcal{A}} u_{n}\right|^{2}-\frac{\sigma}{|y|^{2}}\left|u_{n}\right|^{2}\right)+2 \operatorname{Re} \int_{\mathbb{R}^{N}} \psi_{R} \bar{u}_{n} \nabla_{\mathcal{A}} u_{n} \cdot \nabla \psi_{R}+\int_{\mathbb{R}^{N}}\left|\nabla \psi_{R}\right|^{2}\left|u_{n}\right|^{2},
\end{aligned}
$$

and by the convergence of $u_{n}$ in $L_{l o c}^{2}\left(\mathbb{R}^{N}, \mathbb{C}\right)$, the second and the third term on the right-hand side above tend to 0 if one first lets $n \rightarrow \infty$ and then $R \rightarrow \infty$. We conclude that $\mu_{\infty} \geq 0$.

\section{EXISTENCE OF MINIMIZERS}

We assume that $\Omega=\left(\mathbb{R}^{m} \backslash\{0\}\right) \times \mathbb{R}^{N-m}$ with $2 \leq m \leq N$ and $\mathcal{A}$ is of the form (2.1). We write $(y, z) \in \mathbb{R}^{m} \times \mathbb{R}^{N-m} \equiv \mathbb{R}^{N}$ and consider the problem

$$
\left\{\begin{array}{l}
(-i \nabla+\mathcal{A})^{2} u-\frac{\sigma}{|y|^{2}} u=|u|^{2^{*}-2} u \\
u \in D_{\mathcal{A}, 0}^{1,2}(\Omega, \mathbb{C})
\end{array}\right.
$$

Let $m \leq M \leq N, G$ be a closed subgroup of $O(m) \times O(M-m)$, considered as a subgroup of $O(M)$ in the obvious way, and $\tau: G \rightarrow \mathbb{S}^{1}$ be a continuous homomorphism. Recall that $G \xi:=\{g \xi: g \in G\}$ is the $G$-orbit of $\xi$ and $G_{\xi}:=\{g \in G: g \xi=\xi\}$ its isotropy group. We write $\mathbb{R}^{N} \equiv \mathbb{R}^{M} \times \mathbb{R}^{N-M}$ and assume the following:

$\left(H_{1}\right): \# G \xi=\infty$ for every $\xi \in \mathbb{R}^{M} \backslash\{0\}$.

$\left(H_{2}\right)$ : There exists $\xi^{*} \in \mathbb{R}^{M}$ such that $G_{\xi^{*}} \subset \operatorname{ker} \tau$.

$\left(H_{3}\right): \mathcal{A}$ is $G$-equivariant.

$\left(H_{4}\right): \mathcal{A}(\xi, \zeta)=\mathcal{A}(\xi, 0)$ for all $\xi \in\left(\mathbb{R}^{m} \backslash\{0\}\right) \times \mathbb{R}^{M-m}, \zeta \in \mathbb{R}^{N-M}$.

Hypothesis $\left(H_{2}\right)$ guarantees that $D_{\mathcal{A}, 0}^{1,2}(\Omega, \mathbb{C})^{\tau}$ is infinite dimensional, see Proposition 2.4 . At the end of the section we give some examples of group actions satisfying our assumptions.

Note that the Hardy potential $V(y, z):=\frac{\sigma}{|y|^{2}}$ is $[O(m) \times O(M-m)]$-invariant and, since $m \leq$ $M$, it satisfies $V(\xi, \zeta)=V(\xi, 0)$ for all $\xi \in\left(\mathbb{R}^{m} \backslash\{0\}\right) \times \mathbb{R}^{M-m}, \zeta \in \mathbb{R}^{N-M}$.

The following result is an extension of Proposition 3.2 in [7]. The proof is similar. We give the details for the reader's convenience.

Theorem 3.1. Assume that $\left(H_{1}\right)-\left(H_{4}\right)$ hold, and let $\sigma<\left(\frac{m-2}{2}\right)^{2}$ or $\sigma<\kappa_{s}$ if $\mathcal{A}=$ s $\mathbb{A}$. Then $S_{\mathcal{A}, \sigma}^{\tau}$ is attained. Therefore problem (3.1) has a solution $u \in D_{\mathcal{A}, 0}^{1,2}(\Omega, \mathbb{C})$ which satisfies

$$
u(g \xi, \zeta)=\tau(g) u(\xi, \zeta) \quad \text { for all } g \in G \text {. }
$$

Proof. Let $\left(u_{k}\right)$ be a sequence in $D_{\mathcal{A}, 0}^{1,2}(\Omega, \mathbb{C})^{\tau}$ such that $\left|u_{k}\right|_{2^{*}}=1$ and $\left\|u_{k}\right\|_{\mathcal{A}, \sigma}^{2} \rightarrow S_{\mathcal{A}, \sigma}^{\tau}$. For each $k \in \mathbb{N}$ set

$$
Q_{k}(r):=\sup _{\zeta \in \mathbb{R}^{N-M}} \int_{B_{r}(0, \zeta)}\left|u_{k}\right|^{2^{*}} .
$$

Then $Q_{k}(r) \rightarrow 0$ as $r \rightarrow 0$ and $Q_{k}(r) \rightarrow 1$ as $r \rightarrow \infty$. Hence, there exist $r_{k} \in(0, \infty)$ and $\zeta_{k} \in \mathbb{R}^{N-M}$ such that

$$
Q_{k}\left(r_{k}\right):=\sup _{\zeta \in \mathbb{R}^{N-M}} \int_{B_{r_{k}}(0, \zeta)}\left|u_{k}\right|^{2^{*}}=\int_{B_{r_{k}}\left(0, \zeta_{k}\right)}\left|u_{k}\right|^{2^{*}}=\frac{1}{2} .
$$


Let $w_{k}(\xi, \zeta):=r_{k}^{(N-2) / 2} u_{k}\left(r_{k} \xi, r_{k} \zeta+\zeta_{k}\right)$. Then $w_{k} \in D_{\mathcal{A}, 0}^{1,2}(\Omega, \mathbb{C})^{\tau},\left|w_{k}\right|_{2^{*}}=1,\left\|w_{k}\right\|_{\mathcal{A}, \sigma}^{2}=\left\|u_{k}\right\|_{\mathcal{A}, \sigma}^{2}$ and

$$
\sup _{\zeta \in \mathbb{R}^{N-M}} \int_{B_{1}(0, \zeta)}\left|w_{k}\right|^{2^{*}}=\int_{B_{1}(0,0)}\left|w_{k}\right|^{2^{*}}=\frac{1}{2} .
$$

Passing to a subsequence, we have that $w_{k} \rightarrow w$ weakly in $D_{\mathcal{A}, 0}^{1,2}(\Omega, \mathbb{C})^{\tau}, w_{k}(x) \rightarrow w(x)$ a.e. in $\mathbb{R}^{N}, \quad\left|\nabla_{\mathcal{A}}\left(w_{k}-w\right)\right|^{2}-\frac{\sigma}{|y|^{2}}\left|w_{k}-w\right|^{2} \rightarrow \mu$ and $\left|w_{k}-w\right|^{2^{*}} \rightarrow \nu$ weakly in $\mathcal{M}\left(\mathbb{R}^{N}\right)$. Since

$$
\lim _{k \rightarrow \infty}\left\|w_{k}\right\|_{\mathcal{A}, \sigma}^{2}=S_{\mathcal{A}, \sigma}^{\tau} \lim _{k \rightarrow \infty}\left|w_{k}\right|_{2^{*}}^{2},
$$

using Lemma 2.5 and the definition of $S_{\mathcal{A}, \sigma}^{\tau}$ we obtain

$$
\begin{aligned}
\|w\|_{\mathcal{A}, \sigma}^{2}+\|\mu\|+\mu_{\infty} & =S_{\mathcal{A}, \sigma}^{\tau}\left(|w|_{2^{*}}^{2^{*}}+\|\nu\|+\nu_{\infty}\right)^{2 / 2^{*}} \\
& \leq S_{\mathcal{A}, \sigma}^{\tau}\left(|w|_{2^{*}}^{2}+\|\nu\|^{2 / 2^{*}}+\nu_{\infty}^{2 / 2^{*}}\right) \leq\|w\|_{\mathcal{A}, \sigma}^{2}+\|\mu\|+\mu_{\infty} .
\end{aligned}
$$

Hence

$$
\left(|w|_{2^{*}}^{2^{*}}+\|\nu\|+\nu_{\infty}\right)^{2 / 2^{*}}=|w|_{2^{*}}^{2}+\|\nu\|^{2 / 2^{*}}+\nu_{\infty}^{2 / 2^{*}} .
$$

It follows that one of the quantities $|w|_{2^{*}},\|\nu\|, \nu_{\infty}$ is 1 and the other two are 0. Equality (3.2) implies that $\nu_{\infty} \neq 1$, hence $\nu_{\infty}=0$. Assume $\|\nu\|=1$. Then $w=0$ and $S_{\mathcal{A}, \sigma}^{\tau}\|\nu\|^{2 / 2^{*}}=\|\mu\|$, so $\nu$ is concentrated at the $G$-orbit of a point $\left(0, \zeta^{*}\right)$ with $\zeta^{*} \in \mathbb{R}^{N-M}$ (all other $G$-orbits are infinite because of $\left.\left(H_{1}\right)\right)$. But since $\# G\left(0, \zeta^{*}\right)=1$, (3.2) yields

$$
\frac{1}{2}=\int_{B_{1}(0,0)}\left|w_{k}\right|^{2^{*}} \geq \lim _{k \rightarrow \infty} \int_{B_{1}\left(0, \zeta^{*}\right)}\left|w_{k}\right|^{2^{*}}=\|\nu\|=1 .
$$

This is a contradiction. Therefore $\|\nu\|=0,|w|_{2^{*}}=1$ and $\|w\|_{\mathcal{A}, \sigma}^{2}=S_{\mathcal{A}, \sigma}^{\tau}$.

Theorem 1.1 is just a special case of the previous result with $m=M=N$. In this case, assumption $\left(H_{4}\right)$ is trivially satisfied. Assumption $\left(H_{1}\right)$ holds for the actions of $G=\mathbb{S}^{1}$ or $G=$ $\mathbb{S}^{1} \times \cdots \times \mathbb{S}^{1}$ described in Example 2.3 if, for example, $k=0$ or $k \geq 2$ and $\Gamma=O(k)$ (but not if $k=1$ !). The choices of $\tau$ described there will give solutions with the corresponding symmetry properties (note that $\left(H_{2}\right)$ holds).

For the Aharonov-Bohm potential $s \mathbb{A}$, taking $m=2, M=N, G=\mathbb{S}^{1} \times O(N-2)$ and $\tau_{n}\left(e^{i \vartheta}, g\right)=e^{i n \vartheta}$ in Theorem 3.1 we obtain a solution to problem $\left(\wp_{s}\right)$ satisfying (c) and (d) of Theorem 1.2 when $N \geq 4$. If $N=3$ this $G$ does not satisfy $\left(H_{1}\right)$. However, since $s \mathbb{A}$ does not depend on the last variable, $\left(H_{1}\right)-\left(H_{4}\right)$ hold for $m=M=2$ and $G=\mathbb{S}^{1}$, so we may apply Theorem 3.1 with $\tau_{n}\left(e^{i \vartheta}\right)=e^{i n \vartheta}$ to obtain a solution which satisfies (c). In order to get the other statements of Theorem 1.2 we shall proceed in a different way.

\section{The Aharonov-Bohm potential}

Set $\Omega:=\left(\mathbb{R}^{2} \backslash\{0\}\right) \times \mathbb{R}^{N-2}$. Although the curl of the Aharonov-Bohm potential (1.6) is zero, $\mathbb{A}$ is not the gradient of a function defined on $\Omega$. However, it is the gradient of a function which is defined locally. Namely, if $\theta(y, z)$ denotes the polar angle of $y \in \mathbb{C} \equiv \mathbb{R}^{2}$, then $\nabla \theta=\mathbb{A}$. The function $e^{i \theta}$ is well defined (while $\theta$ is only defined up to an integer multiple of $2 \pi$ ) and a direct computation shows that if $v_{s+n}$ is a solution of $\left(\wp_{s+n}\right)$, then $e^{i n \theta} v_{s+n}$ is a solution of $\left(\wp_{s}\right)$. As 
we did in [7, we use this fact to construct $u_{n}$ by taking $v_{s+n}$ to be a real-valued $\mathbb{S}^{1}$-invariant solution of $\left(\wp_{s+n}\right)$ and setting $u_{n}:=e^{i n \theta} v_{s+n}$. These solutions will have the properties asserted by Theorem 1.2. Since the proof of Theorem 1.2 is similar to that of Theorem 1.3 in [7, we omit some details and concentrate on pointing out the differences.

If $\varphi \in C_{c}^{\infty}(\Omega, \mathbb{C})$ is $\mathbb{S}^{1}$-invariant, i.e. constant on every circle $\left\{\left(y, z_{0}\right):|y|=r\right\}, r>0, z_{0} \in \mathbb{R}^{N-2}$ then, since $\mathbb{A}\left(y, z_{0}\right)$ is tangent to the circle, we have that $\nabla \varphi(x) \cdot \mathbb{A}(x)=0$ for all $x \in \Omega$. Hence,

$$
|\nabla \varphi+i s \mathbb{A} \varphi|^{2}=|\nabla \varphi|^{2}+|s \mathbb{A} \varphi|^{2}
$$

and

$$
\begin{aligned}
\|u\|_{s \mathbb{A}, \sigma}^{2} & =\int_{\Omega}\left(|\nabla u|^{2}+|s \mathbb{A}|^{2}|u|^{2}-\frac{\sigma}{|y|^{2}}|u|^{2}\right) \\
& =\int_{\Omega}\left(|\nabla u|^{2}+\frac{s^{2}-\sigma}{|y|^{2}}|u|^{2}\right) \quad \text { for all } u \in D_{s \mathbb{A}, 0}^{1,2}(\Omega, \mathbb{C})^{\mathbb{S}^{1}} .
\end{aligned}
$$

In this case, the minimizer can be chosen to be real-valued. More precisely, the following holds.

Lemma 4.1. Let $\sigma<\kappa_{s}$ and $G=\mathbb{S}^{1} \times \Gamma$, where $\Gamma$ is a subgroup of $O(N-2)$. Then

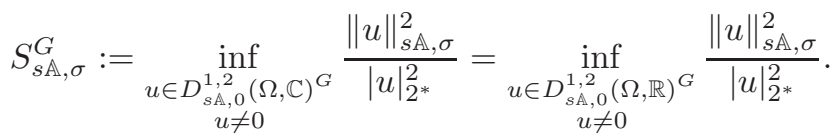

Moreover, if $u$ is a minimizer for the left-hand side, then $|u|$ is a minimizer for the right-hand side.

Proof. Note that $D_{s \mathbb{A}, 0}^{1,2}(\Omega, \mathbb{C})^{G} \subset D_{s \mathbb{A}, 0}^{1,2}(\Omega, \mathbb{C})^{\mathbb{S}^{1}}$. Applying the diamagnetic inequality and (4.2) we obtain

$$
\begin{aligned}
\||u|\|_{s \mathbb{A}, \sigma}^{2} & =\int_{\Omega}\left(|\nabla| u||^{2}+\frac{s^{2}-\sigma}{|y|^{2}}|u|^{2}\right) \\
& \leq \int_{\Omega}\left(|\nabla u|^{2}+\frac{s^{2}-\sigma}{|y|^{2}}|u|^{2}\right)=\|u\|_{s \mathbb{A}, \sigma}^{2}
\end{aligned}
$$

for all $u \in D_{s \mathbb{A}, 0}^{1,2}(\Omega, \mathbb{C})^{G}$ such that $|u|_{2^{*}}=1$. This proves that the right-hand side of (4.3) is smaller than or equal to the left-hand side. Since $D_{s \mathbb{A}, 0}^{1,2}(\Omega, \mathbb{R})^{G} \subset D_{s \mathbb{A}, 0}^{1,2}(\Omega, \mathbb{C})^{G}$ the opposite inequality is obvious. This proves equality (4.3).

If $u$ is a minimizer for the left-hand side of (4.3), then the diamagnetic inequality and equality (4.3) imply that $|u|$ is a minimizer for the right-hand side.

Proof of Theorem 1.2. If $N \geq 4$ we take $M=N$ and $G=\mathbb{S}^{1} \times O(N-2)$, and if $N=3$ we take $M=2$ and $G=\mathbb{S}^{1}$. In both cases we take $\tau \equiv 1$. Then Theorem 3.1] together with Lemma 4.1. gives a real-valued nonnegative minimizer $v_{s}$ for (4.3) such that $\left|v_{s}\right|_{2^{*}}=\left(S_{s \mathbb{A}, \sigma}^{G}\right)^{1 /\left(2^{*}-2\right)}$. It follows from the principle of symmetric criticality [14, 18] that $v_{s}$ satisfies

$$
-\Delta u+\frac{s^{2}-\sigma}{|y|^{2}} u=u^{2^{*}-1} \quad \text { in } \Omega \text {. }
$$

Since $\sigma<\kappa_{s}=: \min _{k \in \mathbb{Z}}|k+s|^{2} \leq s^{2}$, standard regularity results and the maximum principle imply that $v_{s}$ is continuous and $v_{s}>0$ in $\Omega$. If $N=3$ we apply the moving plane method 
[10, 18] to show that $u_{n}(y,-z)=u_{n}(y, z)$ after a translation along the $z$-axis. Therefore $v_{s}$ is $\left[\mathbb{S}^{1} \times O(N-2)\right]$-invariant for every $N \geq 3$. Set

$$
u_{n}:=e^{i n \theta} v_{s+n}, \quad n \in \mathbb{Z},
$$

where as before, $\theta(y, z)$ is the polar angle of $y$. A simple computation shows that

$$
\nabla_{s \mathbb{A}} u_{n}=e^{i n \theta} \nabla_{(s+n) \mathbb{A}} v_{s+n} .
$$

From this, it is easy to see that $u_{n}$ is a solution to $\left(\wp_{s}\right)$, and the statements (a), (c) and (d) hold. If $v_{t}=v_{s}=v$, then

$$
-\Delta v+\frac{s^{2}-\sigma}{|y|^{2}} v=v^{2^{*}-1}=-\Delta v+\frac{t^{2}-\sigma}{|y|^{2}} v,
$$

hence $s^{2}=t^{2}$. So $v_{s} \neq v_{t}$ whenever $s^{2} \neq t^{2}$ which implies (b). Finally, if $t^{2}>s^{2}$, then

$$
\left\|v_{t}\right\|_{t \mathbb{A}, \sigma}^{2}=\left\|v_{t}\right\|_{s \mathbb{A}, \sigma}^{2}+\left(t^{2}-s^{2}\right) \int_{\Omega} \frac{v_{t}^{2}}{|y|^{2}}>\left\|v_{t}\right\|_{s \mathbb{A}, \sigma}^{2}
$$

and therefore $S_{t \mathbb{A}, \sigma}^{G}>S_{s \mathbb{A}, \sigma}^{G}$. Since $\left\|u_{n}\right\|_{s \mathbb{A}, \sigma}=\left\|v_{(s+n)}\right\|_{(s+n) \mathbb{A}, \sigma}$, it follows that (e) is satisfied.

Remark 4.2. Theorem 1.2 holds for $2<p<2^{*}$ if one replaces $(-i \nabla+s \mathbb{A})^{2} u$ by $(-i \nabla+s \mathbb{A})^{2} u+u$. Also Theorems 1.1 and 3.1 with $(-i \nabla+\mathcal{A})^{2} u$ replaced by $(-i \nabla+\mathcal{A})^{2} u+u$, hold for such $p$. The proof that the infimum for an appropriate quotient is attained is similar to that of Proposition 2.3 in [7.

\section{Equivariant Potentials}

Before proving Theorem 1.3 we show how symmetry properties of $\mathcal{A}$ and $\operatorname{curl} \mathcal{A}$ are related.

Proposition 5.1. Let $G$ be a closed subgroup of $S O(N), \Omega$ a $G$-invariant domain in $\mathbb{R}^{N}$ and $\mathcal{A} \in L_{\text {loc }}^{2}\left(\Omega, \mathbb{R}^{N}\right), N \geq 2$.

(i) If $\mathcal{A}(g x)=g \mathcal{A}(x)$ for $g \in G, x \in \Omega$, then $\mathcal{B}(g x)=g \mathcal{B}(x) g^{-1}$.

(ii) If $\mathcal{B}:=\operatorname{curl} \mathcal{A}$ (in the sense of distributions) for some $\mathcal{A} \in L_{\text {loc }}^{2}\left(\Omega, \mathbb{R}^{N}\right)$ and $\mathcal{B}(g x)=$ $g \mathcal{B}(x) g^{-1}$ for all $g \in G, x \in \Omega$, then there exists $\widehat{\mathcal{A}} \in L_{\text {loc }}^{2}\left(\Omega, \mathbb{R}^{N}\right)$ such that $\operatorname{curl} \widehat{\mathcal{A}}=\mathcal{B}(x)$ and $\widehat{\mathcal{A}}(g x)=g \widehat{\mathcal{A}}(x)$.

Proof. (i) If $\mathcal{A}(g x)=g \mathcal{A}(x)$ then, taking derivatives, we obtain $(D \mathcal{A})(g x)=g(D \mathcal{A})(x) g^{-1}$. Hence $(D \mathcal{A})^{t}(g x)=g(D \mathcal{A})^{t}(x) g^{-1}$ and

$$
\mathcal{B}(g x)=(D \mathcal{A})^{t}(g x)-(D \mathcal{A})(g x)=g\left((D \mathcal{A})^{t}(x)-(D \mathcal{A})(x)\right) g^{-1}=g \mathcal{B}(x) g^{-1} .
$$

(ii) If $\mathcal{B}(g x)=g \mathcal{B}(x) g^{-1}$ for all $g \in G, x \in \Omega$, define

$$
\widehat{\mathcal{A}}(x):=\frac{1}{|G|} \int_{G} g^{-1} \mathcal{A}(g x) d g,
$$

where $d g$ is the Haar measure and $|G|:=\int_{G} d g$. Then, for every $g_{0} \in G, x \in \Omega$, we have

$$
\begin{aligned}
\widehat{\mathcal{A}}\left(g_{0} x\right) & =\frac{1}{|G|} \int_{G} g_{0} g_{0}^{-1} g^{-1} A\left(g g_{0} x\right) d g \\
& =g_{0}\left(\frac{1}{|G|} \int_{G} g^{-1} \mathcal{A}(g x) d g\right)=g_{0} \widehat{\mathcal{A}}(x) .
\end{aligned}
$$


Moreover, since

$$
(D \widehat{\mathcal{A}})(x)=\frac{1}{|G|} \int_{G} g^{-1}(D \mathcal{A})(g x) g d g \quad \text { and } \quad(D \widehat{\mathcal{A}})^{t}(x)=\frac{1}{|G|} \int_{G} g^{-1}(D A)^{t}(g x) g d g,
$$

we have that

$$
\operatorname{curl} \widehat{\mathcal{A}}(x)=\frac{1}{|G|} \int_{G} g^{-1} \mathcal{B}(g x) g d g=\frac{1}{|G|} \int_{G} \mathcal{B}(x) d g=\mathcal{B}(x)
$$

as claimed.

Proof of Theorem 1.3. (i) Write $\mathcal{A}(x)=\mathcal{A}_{\tau}(x)+\nu(x) x$ with $\mathcal{A}_{\tau}(x) \cdot x=0, \nu(x) \in \mathbb{R}$. Assume that $\mathcal{A}_{\tau}(x) \neq 0$ for some $x \in \Omega, x \neq 0$. Since $N \geq 3$, we may choose $\gamma \in S O(N)$ such that $\gamma x=x$ and $\gamma \mathcal{A}_{\tau}(x) \neq \mathcal{A}_{\tau}(x)$ (just take $\gamma$ to be an appropriate rotation around the line generated by $x$ ). Then,

$$
\gamma \mathcal{A}_{\tau}(x)+\nu(x) x=\gamma \mathcal{A}(x)=\mathcal{A}(\gamma x)=\mathcal{A}(x)=\mathcal{A}_{\tau}(x)+\nu(x) x
$$

and, therefore, $\gamma \mathcal{A}_{\tau}(x)=\mathcal{A}_{\tau}(x)$. This is a contradiction. It follows that $\mathcal{A}(x)=\nu(x) x$ and, since

$$
\nu(g x) g x=\mathcal{A}(g x)=g \mathcal{A}(x)=\nu(x) g x \quad \text { for all } g \in S O(N),
$$

we have that $\nu(|x|)=\nu(x)$, as claimed. Consequently, $\mathcal{A}(x)=\nabla \varphi(x)$ where $\varphi(x)=\psi(|x|)$ and $\psi(r)$ is a primitive for $\nu(r) r$.

(ii) This is an immediate consequence of (i) and Proposition 5.1

As we have pointed out in the introduction, it follows from Theorem 1.3 that $S O(N)$-equivariant vector potentials can be gauged away if $N \geq 3$ and there is a one-to-one correspondence between radially symmetric solutions to (1.1) and to a similar non-magnetic problem.

Below we shall show by a simple example that radially symmetric solutions to (1.1) may exist also when $G$ is a proper subgroup of $S O(N)$.

Example 5.2. Let $\Omega=\mathbb{R}^{4} \backslash\{0\}$ and

$$
\mathcal{A}(x):=\frac{1}{|x|^{2}}\left(-x_{2}, x_{1},-x_{4}, x_{3}\right) .
$$

Then $\mathcal{A}$ is $S O(2) \times S O(2)$ but not $S O(4)$-invariant and, since curl $\mathcal{A} \neq 0, \mathcal{A}$ cannot be gauged away. Note that, if $u=u(|x|)$, then $\nabla u(x) \cdot \mathcal{A}(x)=0$ and, therefore,

$$
(-i \nabla+\mathcal{A})^{2} u=-\Delta u+|\mathcal{A}|^{2} u=-\Delta u+\frac{1}{|x|^{2}} u,
$$

cf. (4.1). So $u$ is a radial solution to problem (1.2) with $\sigma=0$ iff $u$ solves

$$
-\Delta u+\frac{1}{|x|^{2}} u=|u|^{2^{*}-2} u, \quad u \in D^{1,2}\left(\mathbb{R}^{4}, \mathbb{C}\right)^{S O(4)} .
$$

Theorem 1.1 asserts that a solution to (5.1) exists. 


\section{REFERENCES}

[1] L. Abatangelo, S. Terracini, Solutions to nonlinear Schrödinger equations with singular electromagnetic potential and critical exponent, J. Fixed Point Theory Appl. 10 (2011), 147-180.

[2] G. Arioli, A. Szulkin, A semilinear Schrödinger equation in the presence of a magnetic field, Arch. Rational Mech. Anal. 170 (2003), 277-295.

[3] M. Badiale, G. Tarantello, A Sobolev-Hardy inequality with applications to a nonlinear elliptic equation arising in astrophysics, Arch. Ration. Mech. Anal. 163 (2002), 259-293.

[4] J. Chabrowski, A. Szulkin, M. Willem, Schrödinger equation with multiparticle potential and critical nonlinearity, Topol. Meth. Nonl. Anal. 34 (2009), 201-211.

[5] S. Cingolani, M. Clapp, Intertwining semiclassical bound states to a magnetic nonlinear Schrödinger equation, Nonlinearity 22 (2009), 2309-2331.

[6] S. Cingolani, M. Clapp, and S. Secchi, Multiple solutions to a magnetic nonlinear Choquard equation, $Z$. angew. Math. Phys. 63 (2012), 233-248.

[7] M. Clapp, A. Szulkin, Multiple solutions to a nonlinear Schrödinger equation with Aharonov-Bohm magnetic potential, Nonlinear Differ. Equ. Appl. (NoDEA) 17 (2010), 229-248.

[8] T. том Dieck, "Transformation groups", Walter de Gruyter, Berlin-New York 1987.

[9] L.C. Evans, R.F. Gariepy, "Measure theory and fine properties of functions", Studies in Advanced Mathematic, CRC Press, Boca Raton, FL 1992.

[10] B. Gidas, W.-M. Ni, and L. Nirenberg, Symmetry of positive solutions of nonlinear elliptic equations in $\mathbb{R}^{N}$, in "Mathematical analysis and applications", Part A, pp. 369-402, Adv. in Math. Suppl. Stud. 7a, , Academic Press, New York-London, 1981.

[11] A. Laptev, T. Weidl, Hardy inequalities for magnetic Dirichlet forms, in "Mathematical results in quantum mechanics" (Prague, 1998), pp. 299-305, Oper. Theory Adv. Appl. 108, Birkhäuser, Basel, 1999.

[12] E.H. Lieb, M. Loss, "Analysis", Graduate Studies in Math. 14, Amer. Math. Soc. 1997.

[13] V.G. MAZ'JA, "Sobolev Spaces", Springer-Verlag, Berlin-Heidelberg 1985.

[14] R. Palais, The principle of symmetric criticality, Comm. Math. Phys. 69 (1979), 19-30.

[15] S. Secchi, D. Smets, M. Willem, Remarks on a Hardy-Sobolev inequality, C. R. Math. Acad. Sci. Paris 336 (2003), 811-815.

[16] A. Szulkin, S. Waliullah, Sign-changing and symmetry-breaking solutions to singular problems, Complex Var. Elliptic Equ. 57 (2012), 1191-1208.

[17] S. Waliullah, Minimizers and symmetric minimizers for problems with critical Sobolev exponent, Topol. Meth. Nonl. Anal. 34 (2009), 291-326.

[18] M. Willem, "Minimax theorems", PNLDE 24, Birkhäuser, Boston-Basel-Berlin 1996.

Instituto de Matemáticas, Universidad Nacional Autónoma de México, Circuito Exterior, C.U., 04510 MÉxico D.F., Mexico

E-mail address: mclapp@matem.unam.mx

Department of Mathematics, Stockholm University, 10691 Stockholm, Sweden

E-mail address: andrzejs@math.su.se 